\title{
Urinary System Findings Time Point Reference
}

National Cancer Institute

\section{Source}

National Cancer Institute. Urinary System Findings Time Point Reference. NCI Thesaurus.

Code C162318.

The point in time that acts as a fixed reference point to a urinary system finding. 